\title{
Urgences
}

\section{Les Nuits de Vitre - La nuit des masques}

Paul-Émile Saulnier et Manon Regimblad

Numéro 26, décembre 1989

Des textes qui chantent

URI : https://id.erudit.org/iderudit/025560ar

DOI : https://doi.org/10.7202/025560ar

Aller au sommaire du numéro

Éditeur(s)

Urgences

ISSN

0226-9554 (imprimé)

1927-3924 (numérique)

Découvrir la revue

Citer ce document

Saulnier, P.-É. \& Regimblad, M. (1989). Les Nuits de Vitre - La nuit des masques. Urgences, (26), 101-116. https://doi.org/10.7202/025560ar d'utilisation que vous pouvez consulter en ligne.

https://apropos.erudit.org/fr/usagers/politique-dutilisation/ 
Le règne des fins... les deux figures contrastées du pouvoir souverain et du martyre qui représentent l'histoire. C'est pourquoi il n'y a pas d'eschatologie baroque; mais c'est précisément pour cette raison qu'il y a un mécanisme du monde, où toutes les existences terrestres sont rassemblées et exaltées, avant d'être livrées à leur fin.

Walter Benjamin, Origine du drame ba roque allemand

(cité par Christine Buci-Glucksmann: La Folie du voir)

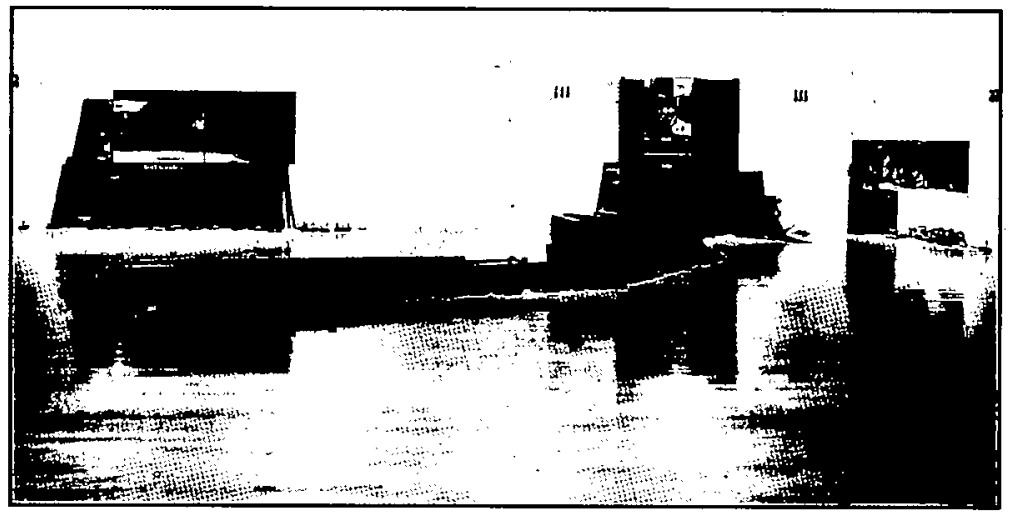

Vue partielle de l'exposition Les Nuits de Vitre présentée à la galerie Expression de StHyacinthe, 1989

Photo: Pierre Groulx

Paris et Berlin - deux villes qui sont comme des coups de poing au sol, taillées et sculptées au fil des siècles. Paris et Berlin - deux villes dont les cathédrales s'amusent à diviser la lumière. Comment raconter ces villes: la turbulence de leur vie quotidienne, l'achalandage des quartiers, la beauté architecturale des édifices, des places publiques et des monuments, de la charge historique contenue...

\section{Paris}

Du Grand au Petit Palais bien assis l'un en face de l'autre, du Musée d'Orsay au Musée du Louvre, autre complicité parallèle d'une Seine dont les rives se chamaillent un même public; c'est en faisant le parcours entre 1'Opéra et Notre-Dame de Paris que me vint l'idée de regrouper mes interventions sous le couvert d'un opéra visuel intitulé: Les Nuits de vitre.

1985 - Voyage à Berlin

Allemagne... pays divisé.

Berlin ... ville divisée... une moitié emmurée. 
Trois corridors routiers et trois corridors aériens pour y avoir accès.

Berlin... un territoire occupé.

À proximité du mur, les routes rétrécissent, les fouilles se font, les papiers passent d'une main à l'autre. Les vérifications n'en finissent plus: l'attente, la méfiance, l'angoisse. Les miroirs chercheurs roulent et rampent sous les véhicules: soupçons. Deuxième arrêt: les questions reprennent. Je passe. Allemagne de l'Est. Une autoroute: le temps est mesuré. Les hélicoptères patrouillent, surveillent: SURVEILLANCE. Char d'assaut: étrange monument-chien de garde dont le canon accusateur pointe Berlin Ouest. Banderole: écriture provocatrice - «Berlin Ouest capitale de l'Allemagne de l'Est». En bout de ligne, le passage «s'engrille», s'entoure de clôtures, s'étire et s'effile. Miradors. Mitrailleuses. Berger allemand en laisse. Les questions reprennent: contrôle. Le MUR. Il y a là un mur qui entoure, étouffe et enserre. Blanc d'un côté, gris de l'autre. Le terrain est malsain et dangereux. No man's land! Les voix murmurent. Interdiction! Cachez vos caméras. Cachez vos lunettes d'approche. Ici les gens ont peur. Ici les gens craignent. Et puis finalement... j'allais dire presque bêtement... je passe. Welcome to West Berlin - You are entering the American Sector!

\section{Berlin Ouest}

Îlots dans l'Allemagne de l'Est. Prime de risque et d'isolement pour les ouvriers. Néons: en plus de l'allemand, ici on parle le français et l'anglais.

Une cathédrale figée par ses blessures. Un métro lourd, fatigué de ses témoignages. Promenade le long d'un mur. Poésie meurtrie. D'étranges monuments Mémorial de Plötzensee: deux petites chambres grises et tristes comme la mort témoignent de l'élimination des opposants au régime nazi. Il y a bien eu ici un adolescent allemand exécuté!

Visite à Berlin Est! Il faut repasser le mur. Checkpoint Charlie! Ici les soldats sont décontractés, sourient même. Et puis le passage (le couloir) rétrécit. Contrôle sur contrôle. Vérification des passeports. Autorisation de 24 heures. L'argent est contrôlé, limité à 25 DM et pèse peu! Qu'allezvous faire à Berlin Est? Visité... vous savez... tourisme... d'une chambre à l'autre... ça y est... Berlin Est.

\section{Berlin Est}

Sur un mur la photo d'un groupe d'ouvriers. Immersion du citoyen ouvrier dans le régime. Gratification: ici on parle l'allemand et on parle le russe. La place publique est déserte. La porte de Brandebourg... fermée jusqu'à nouvel ordre. Prenez garde aux émissions de télévision que vous écoutez et regardez. Oncraint les vents de l'Ouest. Reconstruction lente. Cliquetis des caméras. La journée se vit. Les choses se découvrent. L'heure! Il est 
temps de revenir à autre chose. Le Mur... rien à déclarer? Non! Et puis... have a good night vous crie l'impérial isme américain. Journée émouvante. Nuit de ville. Laissons le mur agiter ses dessins.

Un mur! Une idée pour déchirer un pays. Un mur! Une erreur, une gifle à la démocratie, un affront à l'humanité toute entière.

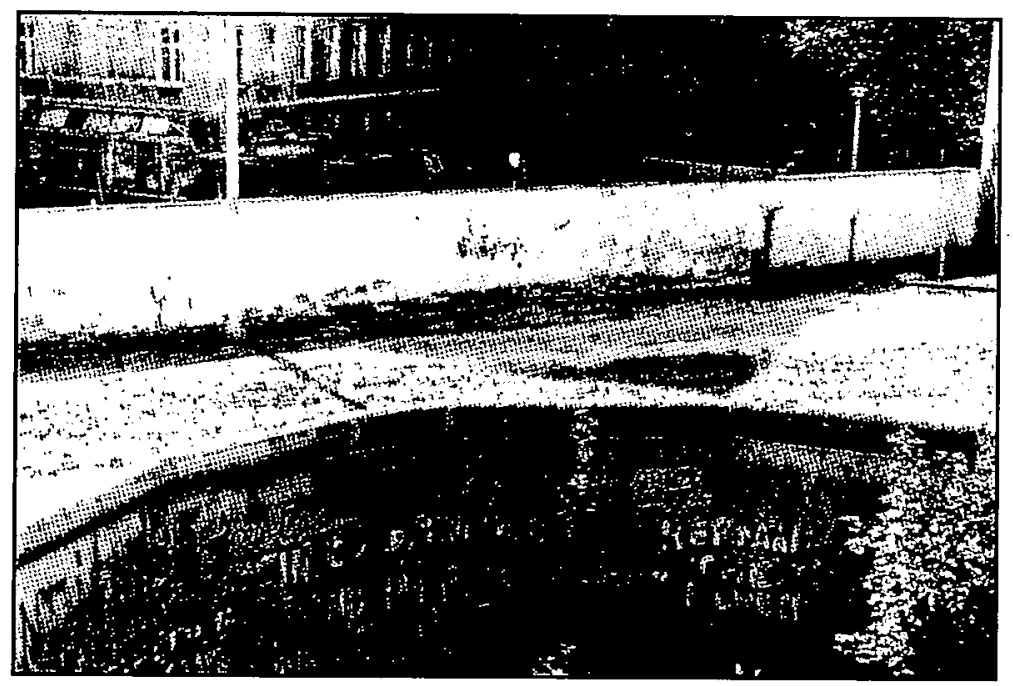

Le Mur (Berlin)

Photo: Lucie Côté-Saulnier

\section{Opera}

Les Nuits de vitre

La Seconde Guerre mondiale sert de toile de fond à une image qui veut témoigner de l'horreur de ce temps-là et dresser un tableau de l'horreur de notre temps. Depuis plusieurs années, une grande partie de mon travail de recherche porte sur la ville de Berlin, sur son statut et sur son histoire pour atteindre un lieu dépassant Berlin afin de rejoindre la situation européenne et la scène internationale de ce temps-là et de notre temps. L'image s'écrit tantôt par le dessin, tantôt par la réalisation d'installations dans lesquelles le dessin est amené à cohabiter avec la sculpture, mettant ainsi en interaction un double espace. Le dessin et les installations (cumulatives, éclatées et fragmentées) explorent l'espace (réel, fictif, représenté et évoqué), le mouvement, le temps, le lieu (l'interne, l'externe, la vie, la mort). L'idée de comparer le IIIe Reich à un vaste opéra est venue à Paris en 1987. 


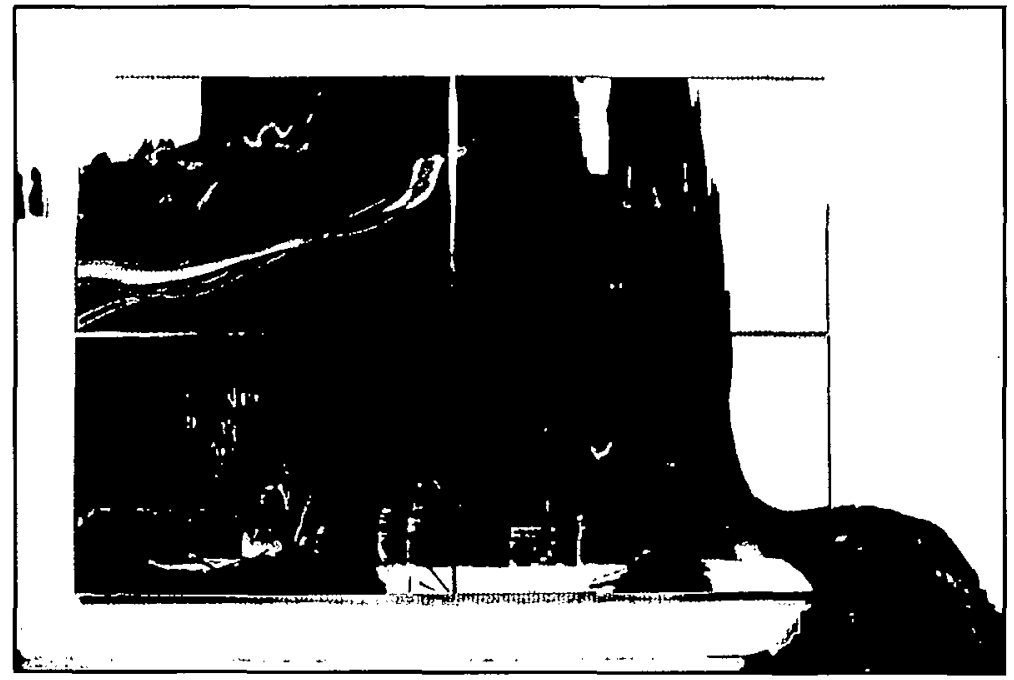

La Nuit des Masques - 1989

$240 \times 150 \mathrm{~cm}$

Papier somerset, fusain, acrylique, pastels, toile

Photo: Lucie Côté Saulnier

Opera

La Nuit des masques

Que la scène baroque trouve dans l'opéra et la peinture ses métaphores, qu'elle s'instituedans une folie du voir qui affecte tous les Arts et Savoirs de l'époque, ets'énonce dans les grands traités de rhétorique italiens ou espagnols du dix-septième siècle; tel est l'enjeu de cette Archéologie du Regard baroque, un Regard du fantasme, un regard anamorphique tissé d'amour et de mort, de rien et de sublimité, de mélancolie et de *furore*. *Là, dans cette Raison insuffisante, dans cette érotique flamboyante, l'on pense avec les yeux. *

Christine Buci-Glucksmann

L'opéra "Les nuits de vitre» prélude à l'opéra "La Nuit des masques». Il évoque les opérations du même théâtre, c'est-à-dire un siècle éventré par les jeux de pouvoir, de puissance, de déchirement, de domination, d'autorité et d'opposition. L'opéra, par La Nuit des masques, reflète l'absurde et la cruauté de ces jeux par la mascarade, le déguisement, le camouflage, la fiction et le grotesque: bals et défilés.

L'extase, l'exaltation, la raillerie, la solitude, la révolte, la passion, la barbarie... l'enivrement de la vie à la terreur dénomme, définit l'être du XXe siècle. 
Les * gargouilles

Je les prends à témoin!

... burlesques et cornues, anges ou démons, monstres et dragons, reptiles rampants, bêtes fauves, oiseaux, béliers... depuis des siècles elles observent le monde du haut de leurs perchoirs: cathédrales et tours, palais et châteaux. Elles grimacent. Elles regardent les hommes vivre. Elles crachent par jour de mauvais temps. Peut-être crachent-elles par lassitude, par révolte, par impatience ou par mauvaise habitude. Elles sont-là, les griffes bien accrochées dans la pierré, s'étirant et s'avançant dans le vide pour mieux voir, pour ne rien manquer des histoires. Elles sont là défiantes, arrogantes et insolentes - spectatrices mal léchées, fières et méprisantes -, elles sont là fidèles à leur poste et à leur fonction, bossues et crochues, malsaines et enjôleuses, se tordant, ouvrant l'anus bien grand comme un réceptacle pour filtrer une eau qui leur entre (pénètre) par tout le corps et qu'elles régurgitent en toute hâte.

Et la nuit... la nuit, alors que les hommes dorment, les toitures s'animent et les ombres s'agitent. Les gargouilles se livrent d'étranges combats. Et si par hasard il vous arrive de ne pas fermer l'oeil de la nuit... si vous tendez l'oreille, vous entendrez leurs ricanements et sentirez les bâtisses trembler. 


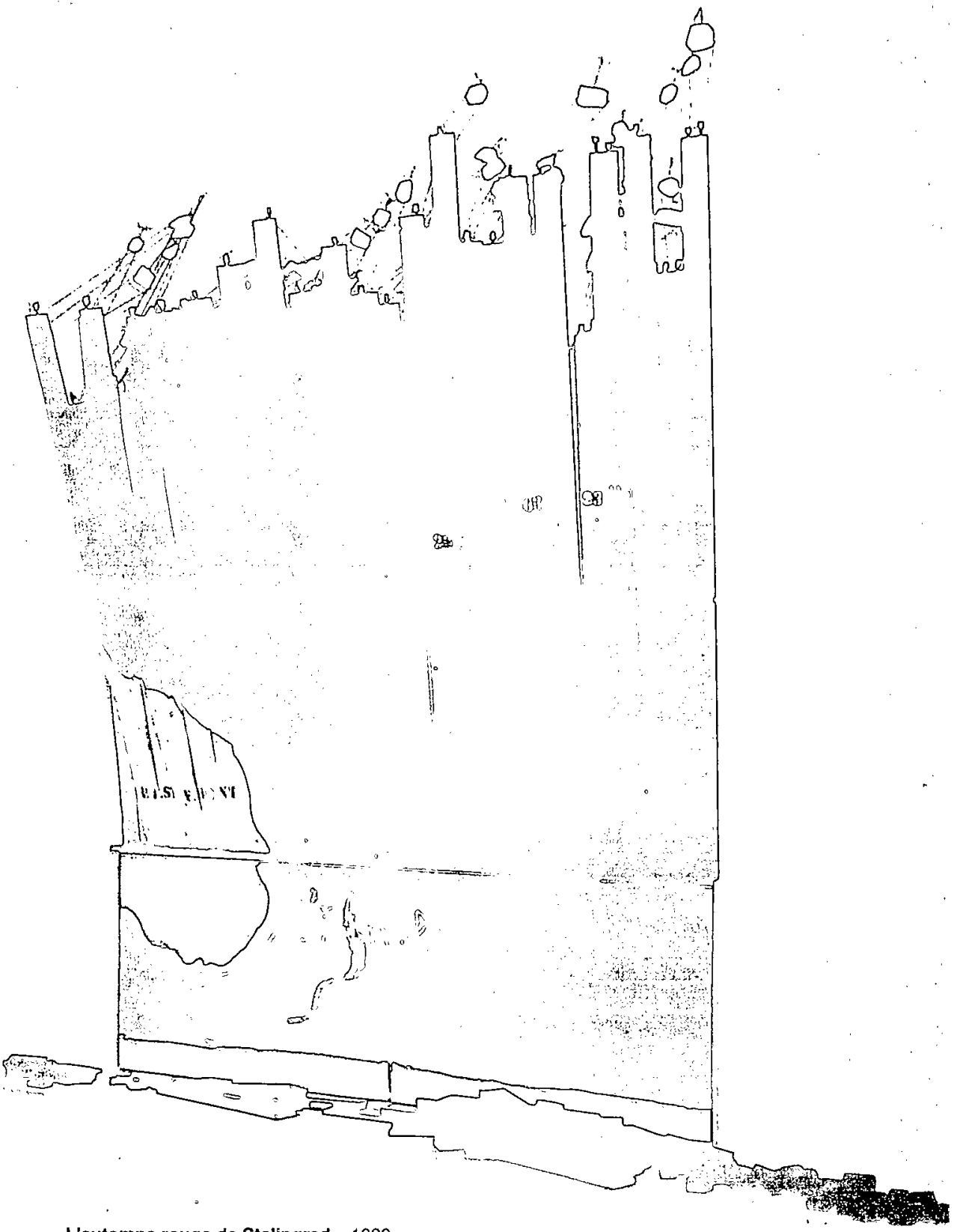

L'automne rouge de Stalingrad - 1989

$210 \times 360 \times 50 \mathrm{~cm}$

Papier somerset, bois, fusain, acrylique, pastels, corde

Photo: Pierre Groulx 


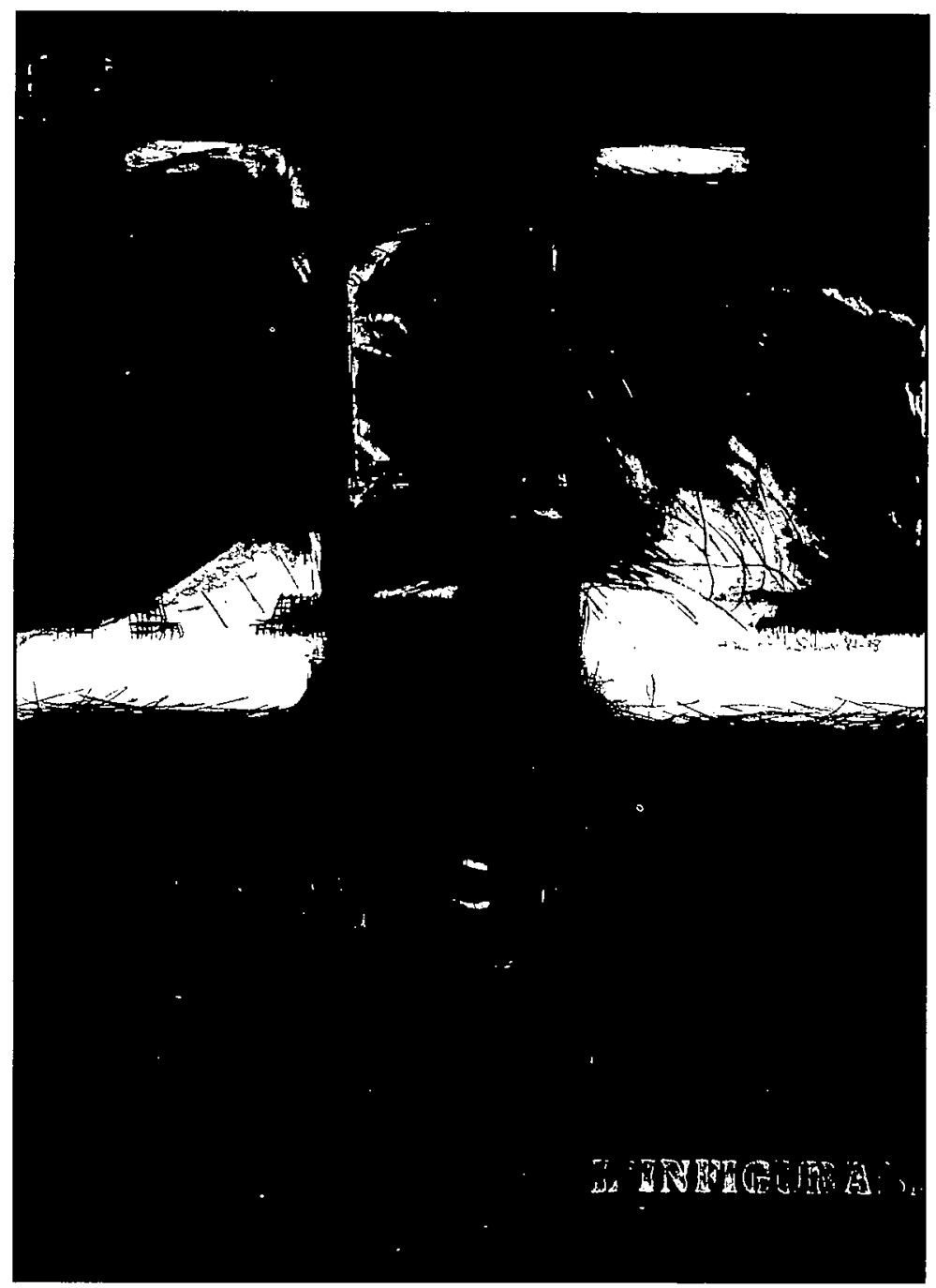

L'INFIGURABLE - 1986-1988 $105 \times 160 \mathrm{~cm}$ Papier somerset, acrylique, fusain, pastels 


\section{Derrière Les Nuits de Vitre - La Nuit des masques}

\section{LES NUITS DE VITRE - LA NUIT DES MASQUES}

Oeuvre installative présentée par Paul-Émile SAULNIER, en 17 séquences: 1 - Sans titre (1985-87); 2- Sans titre (1988); 3-Calmez ces démons (1988); 4-Crématorium (1989); 5 Berlin (1986); 6- Les nuits de vitre (1988); 7- Jardins de cendre (1987); 8- Sans titre (198488); 9- L'infigurable (1985-1988); 10- Cri (1984); 11 - A matter of war (1986); 12-Stupid game (1986); 13- Cri 2 (1984); 14- Strange writings about Berlin (1988); 15- Eclipse (1988); 16- L'automne rouge de Stalingrad (1989); 17- La nuit de cristal (1988).

Dimensions approximatives du lieu d'accueil:

largeur: 40 pieds

longueur: 60 pieds

hauteur: 12 pieds

Allons à l'opéra. Retournons aux nuits vitrées. Tout de suite.

Au commencement une certaine inquiétude trouble les lieux. Emporté(e)s par le champ sculptural, les spectateurs, fragiles, éprouvent l'inconstance du monde. Le corps se perd. L'oeil s'égare. Il se frappe à l'insondabilité des caisses refermées. Eccartante, l'oeuvre nous échappe. Nous apparaissons de plus en plus fragiles. Au bord du gouffre, le regardant est perdu.

13h30:

Rimouski, le 3 novembre 1989

Cégep de Rimouski

Conférence:

Que sont devenus les Allemands?

Dieter F. Rauch, journaliste culturel à Berlin.

Alors même que le mur de la honte s' apprêtait à tomber, que les réalités géographique, politique, culturelle des Europes se transformaient d'heures en heures, l'événement Les Nuits de vitre - La nuit des masques s'enclenchait, à Rimouski, par la conférence de Dieter, arrivant directement de Berlin Ouest.

17h00:

Musée régional de Rimouski

Concert d'Erich Kory

Lecture publique de textes de Marie-Andrée Massicotte et Lucien Cimon par les aureurs, de Paul Chanel Malenfant par Elisabeth Haghebaert.

Dans la pénombre du lieu, trois lutrins noirs au-dessus desquels sont suspendues autant de lumières, petits phares de la nuit démasquée. Autant d'interprètes à prendre la parole. Au verbe s'épanchant s'emmêle la poésie musicale du violoncelliste Erich Kory. Alors que les fragments violonés au mur et au sol s'entêtent dans leur silence, le violoncelliste entame son improvisation. 
Le phrasé musical comporte son lot de mesures classiques. La longue fréquentation de Bach par le musicien contribue à l'enrichissement de la joute improvisée. Les arrangements classiques s'accouplent aux formes jazzées. Par moment gémissants ou emportés, ils se lient aux accents tziganes et yiddish. Le frémissement des cordes, leurs vibrantes élongations, leur diversité vibratoire incisive, mordante, saccadée, vive et lancinante plongent à la fois le verbe et l'image dans l'abîme insondable de la représentation. L'usage de l'électroacoustique réverbère la voix du violoncelle, ajoutant à la tessiture de l'instrument. La musique sérielle reprend cette économie de la répétition à l'oeuvre dans l'opéra visuel.

Dans la solennité du moment, autour des caisses vidées, entre les planches entassées, les amoncellements innombrables des petits paquets de journaux solidement lacés, aux côtés des cordes (dé)liées, par delà les murs divisionnaires se recueillent des spectateurs, touchés par les accords du violoncelliste. Par l'étreinte de la parole prise.

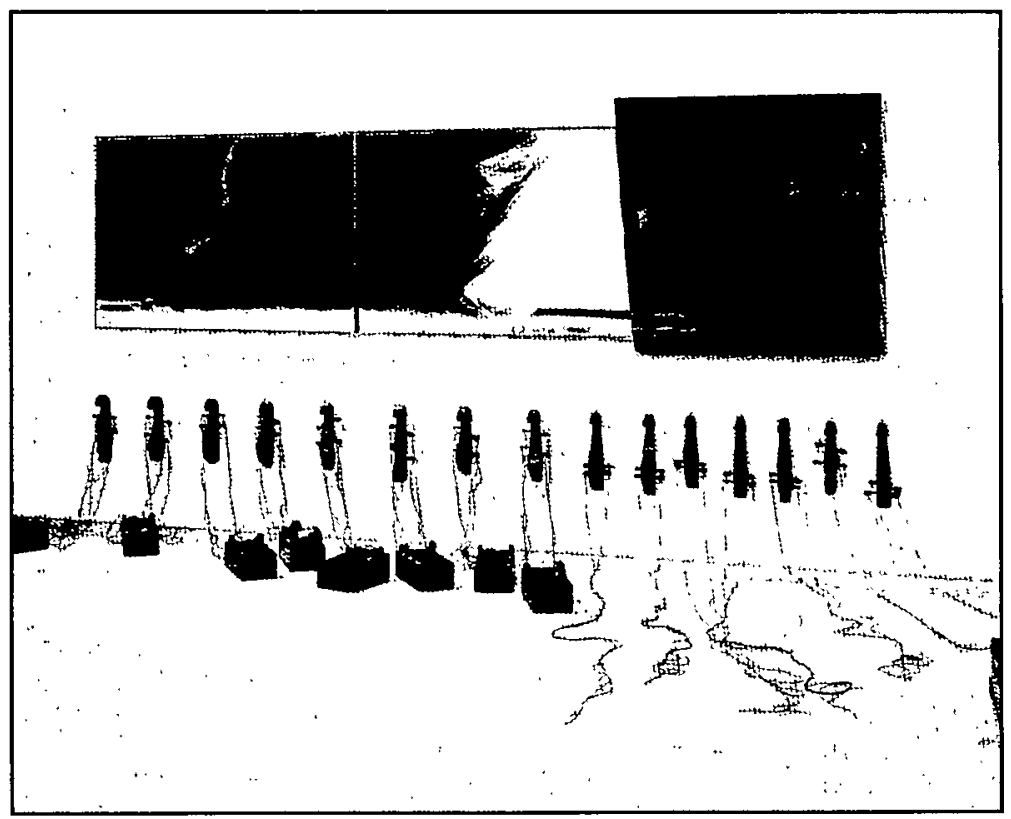

Berlin - 1986

$240 \times 70 \times 40 \mathrm{~cm}$

Fusain, acrylique, carton ondulé, papier somerset, bois, pastels

Photo: Pierre Groulx 
Peut-être n'y a-t-il rien à redire, l'oeuvre seule nous permettant d'accéder à cet Infigu rable. Et pourtant, je risque cet écart de librettiste. Emballée. À nouveau éprise. L'oeil collé à la fenêtre, le regard se fait discriminateur. Il éclaire. Le corps s'y conjugue. L'attribution perceptuelle reconstruit le lieu. L'oeuvre nous donne à voir. Singulièrement, le corps regardant éclaire à son tour, malgré la nuit noire. Il nous rend l'opéra. À notre façon.

De nouveau touchée. Écorchée vive. Profondément endeuillée. Nous voilà atteints.

Nous retrouvons l'écriture plastique familièreà Saulnier. Sa composition en frise s'étale. L'installation s'emploie à une rénovation du monde des objets pendant que par terre et au mur se ramassent les fragments violonés, les planches, les cordes, les ficelles, les journaux pliés. Désamorcées, les trappes à souris voisinent avec les tapons journalistiques résolument illisibles.

Or il y a trop à dire. Il me faudrait m'arrêter et me pencher attentivement sur chacun des fragments exposés. L'un conduit à l'autre. Les autres se ramènent à l'un. Faut-il le rappeler, Saulnier pratique un art de l'enrobement, emboîtant tour à tour l'art et ses histoires. Reliant chacun de ses empaquetages à un réseau citationnel. Des regardants, familiers de son oeuvre, retracent ces repères qui jalonnent l'installation, au fil de ses itinérances. En fait, chacun des éléments compositionnels s'inscrit dans un vaste système de renvois autoréférentiels.

Chacune des pièces violonées, chacune des boîtes, les planches, les cordes assurent la continuité plastique irréférentielle.

L'oeuvre se déballe. Des caisses se sont extirpées les nocturnes vitrées. Mises en boîtes, les pièces sont décaissées, le temps d'un transit. Mises en pièces, elles seront rencaissées. Saulnier nous a habitué au déballement intégral de ces installations migratrices.

Les Nuits de vitre - La Nuit des masques bénéficient d'une complicité rare avec leur lieu d'installation, s'étalant sur deux étages du musée. L'enveloppe architecturale englobe chacune des séquences. Leur solidarité conceptuelle nous empêche de les isoler inutilement. La portée de l'opéra s'épand sous le regard. Sous l'oeil attentif l'oeuvre se délie. Elle circule dans l'espace édifié. Entre le bâtiment et l'installation s'accomplit une connivence peu souvent égalée. À vrai dire, une sorte d'osmose s'établit. 


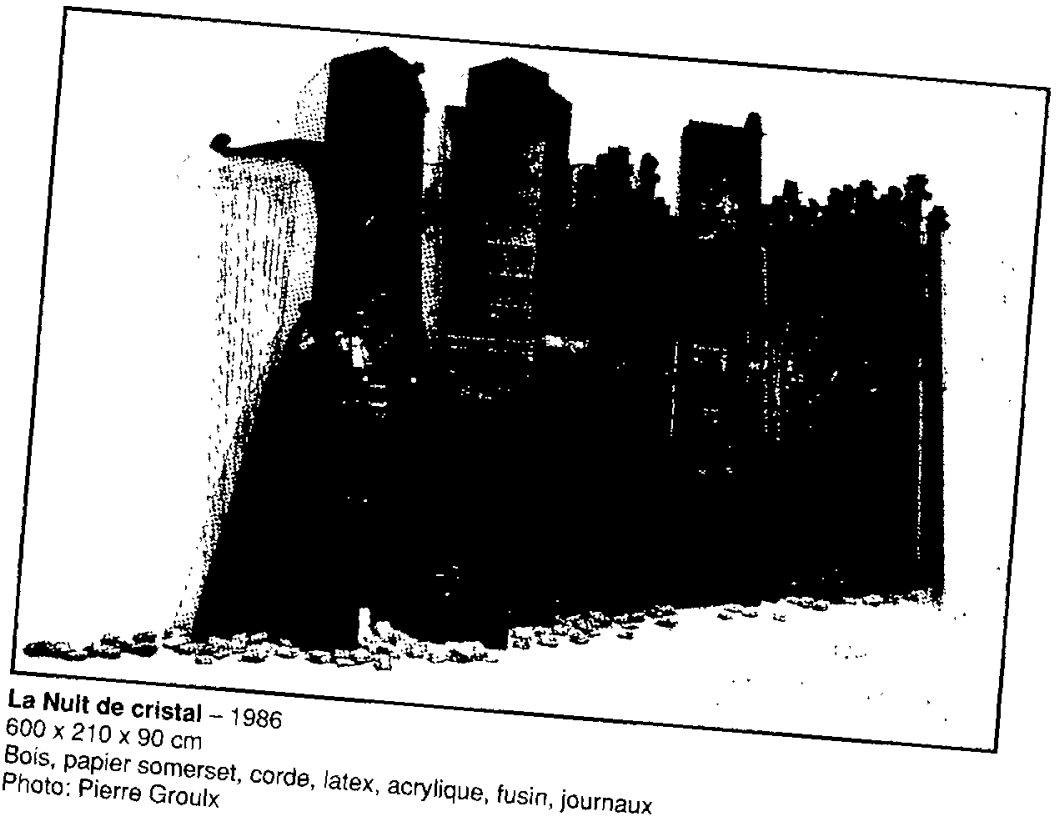

\section{1}

\section{LES NUITS ROMANES}

sculpture est envahissante. Sans romanes, l'opéra nous dépasse. $L_{a}$ rappelée. La structure compositionnelle de la plastique médiévale est de vitre - La Nuit des masques à leur ins l'art roman trame Les Nuits principes qui régissent la sculpture teur insu. À la lumière des principaux semble souscrire à ces règles: par exomane, l'ouuvre de Saulnier, en partie, C'est-à-dire qu'ici l'oeuvre, sans se somple, à la primauté de l'architecture'. ture, $s^{\prime} y$ appuie solidement. L'architecture définitivement à l'architecvre se développe selon les limites artecture emboîte l'installation. L' s'étirent, s'allongent. Elles suivent le bâtiment. Le fordes, les planches
remarqué dans la sculptures primordiales d'a sculpture romane - la génération armalisme interne interne ouen autres formes, comme une sorte de partir des formes dans Les Nuits de vinme une «logique de la métamorphaine analogique elle-même reprend l'idé - La Nuit des masques. $\grave{A}$ partir - est présent séquences. Les dével idée de l'enveloppe architecturale), se da boîte (qui des petits paque une chaîne regets de journaux, des fragments violonés shes, des cordes, dessinées, elles veitlent et se raccordententre eux. Ques s'inscrivent dans Devantl'occupation des territoires de la représentation.
à l'ampleur de l'oérgouilles à l'ampleur de l'oeuvre. Désemparé(e)s. 
Inflationniste, la portée de l'installation s'étend. Déballé, le corps de l'oeuvre nous entraîne dans sa démesure vertigineuse.

Or la sévérité et la sobriété de la plastique médiévale contiennent l'expansion installative. Le calme monastique se prête au pélerinage. La lumière feutrée empreint l'atmosphère d'une solennité formelle. Au ras du sol surgit l'éclairage. Tandis que, de leurs lumières, les miradors balaieraient le sol, à l'inverse l'éclairage, à partirdu sol des Nuits de vitre, s'élève dans l'espace. Ainsi, moinscrue, la blancheur des murs d'accueil s'atténue. L'accent se porte vers l'image sculpturale.

La sérénité du lieu sacre l'opéra. L'austérité des planches, conjuguée à la noirceur apparente, tombe sur le spectateur.

\section{LA CAISSE}

La monstration du rien emboîté, l'encaissement de la pure perte se heurtentà l'indicibilité. Les caisses retranchent à la visibilité apparente le leurre référentiel, consacré.

Or l'emboîtement convie la mémoire au souvenir, à l'acte remémorant. Commémorative, la boîte réveille. Elle déplie une mémoire vagabonde. Des petits sarcophages aux entrailles ouvertes (Berlin), aux nombreuses caisses-monuments de la Stupid game - vaste cimetière roulant prenant des allures de train de la mort -, les caisses révèlent leurs rappels.

La mise en boîte monumentale de l'innen et de l'aussen nous confronte à l'échappement du temps. Empaqueteur, l'artiste emmure le vide. Il emballe l'espace. Il le met en boîte. Les planches l'emballent, contraignant spatio-temporellement le lieu del'installation. Leur référentialité nous est retirée. Les référents sont déniés. Le visible actuel, empirique, ne se rapporte pas à un invisible factuel comme un objet se déroberait à la vue, caché derrière un autre. Mais ce sont des relations enveloppements, encaissements, dérobements - à la chaîne qui font l'objet de références.

Empaqueté, l'indicible se transforme en signe. Trocable, échangeable, la misère des uns fait l'objet d'un commerce prospère. Commerce politique. Idéologique. Pourtant la marchandise emballée nous résiste. Elle ne tolère plus d'être livrée. Forte de son itinérance, l'installation a appris à résister. 


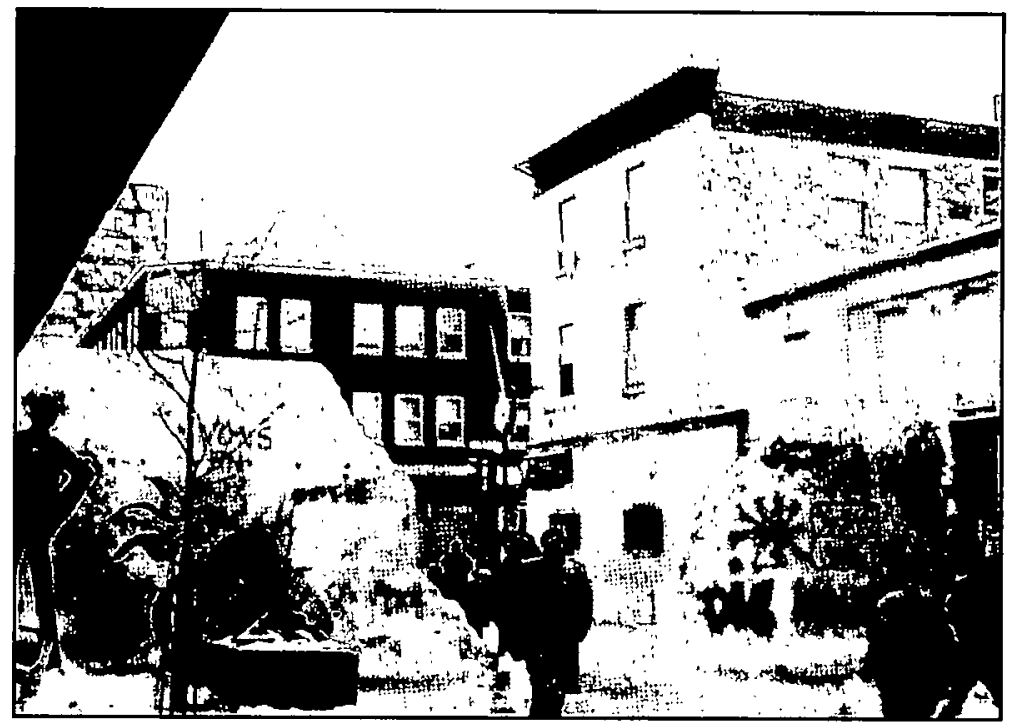

Photo: Manon Regimbald

Oeuvre réalisé par des inconnus, rue Prince-Arthur, Montréal, 7 décembre 1989. En épitaphe: Dédié aux quatorze victimes de Polytechnique, 6 décembre 1989.

Montréal, le 11 décembre 1989

Alors même que là-bas s'effondre le mur [un mur], que tombent desfrontières, voilà que le 6 décembre entache notre blanc hiver. Par quatorze fois. La neige est rougie. Notre société rougissante. D'un mur à l'autre subsistent les haines, le mépris séculaire et patriarcal, la misogynie démesurée d'une société, assoiffée de domination. Le commerce de la violence - de l'intimidation quotidienne aux actes spectaculaires -, prévaut, voire est encouragée. La complicité des pouvoirs religieux, législatifs et judiciaires en légitimise trop souvent l'existence. Par leur indifférence, leur ignorance crasses.

Ce n'est pas sans raison que certains ont voulu (cru?) ignorer cette réalité sous les épithètes neutres de jeunes victimes, d'étudiants, d'enfants, croyant taire du même coup ce gynécide.

Dans leur aria monumentale, Les Nuits de vitre rappellent. Elles réveillent l'histoire. Leur noirceur réfléchit la représentation. Sous la folle accumulation des petits paquets de journaux étroitement ficelés, réduits 
à l'illisibilité, des Nuits de vitre - La Nuit des masques, derrière l'expression de la censure, de l'information muselée, contrôlée, manipulée s'entassent la mémoire des femmes, tue.

Geneviève Bergeron (1968-1989)

Hélène Colgan (1966-1989)

Nathalie Croteau (1966-1989)

Barbara Daigneault (1967-1989)

Anne-Marie Edward (1968-1989)

Maud Haviernick (1960-1989)

Barbaria Maria Klueznick (1958-1989)

Maryse Laganière (1964-1989)

Maryse Leclair (1966-1989)

Anne-Marie Lemay (1962-1989)

Sonia Pelletier (1961-1989)

Michèle Richard (1968-1989)

Annie St-Arneault (1966-1989)

Annie Turcotte (1968-1989)

Le visible est troqué. La noirceur des nocturnes vitrées s'accomplie et l'obscurcit. Une fois emballé, l'invisible des Nuits de vitre - La Nuit des masques s'accomplit.

\section{L'ENCAISSEMENT}

Rendre présent l'infigurable. Qu'on ne s'y trompe point. L'infigurable ne se cantonne pas à la $9 \mathrm{e}$ séquence. Il se cache sous chaque planche. Chaque corde l'encense. Les journaux le défigurent. Noircis. Obscurcis. Charbonnés. Incinérés. Censurés. Sous des airs représentationnels, la vitrine installative en démasque l'inaccessibilité. Vitré, l'infigurable, dans un élan révélateur, exhibe son incapacité à figurer, ironiquement. Embô̂té, cordé, plié, emmuré, l'insondable s'abìme derrière Les Nuits de vitre - La Nuit des masques.

\section{Manon Regimbald}

Du 3 novembre au 17 décembre 1989, au Musée régional de Rimouski.

Des tranches de cet opéra visuel seront présentées à la galerie Skol de Montréal en mars 1990.

1 Henri Focillon, L'art des sculpteurs romans, Paris, PUF, 1964. 


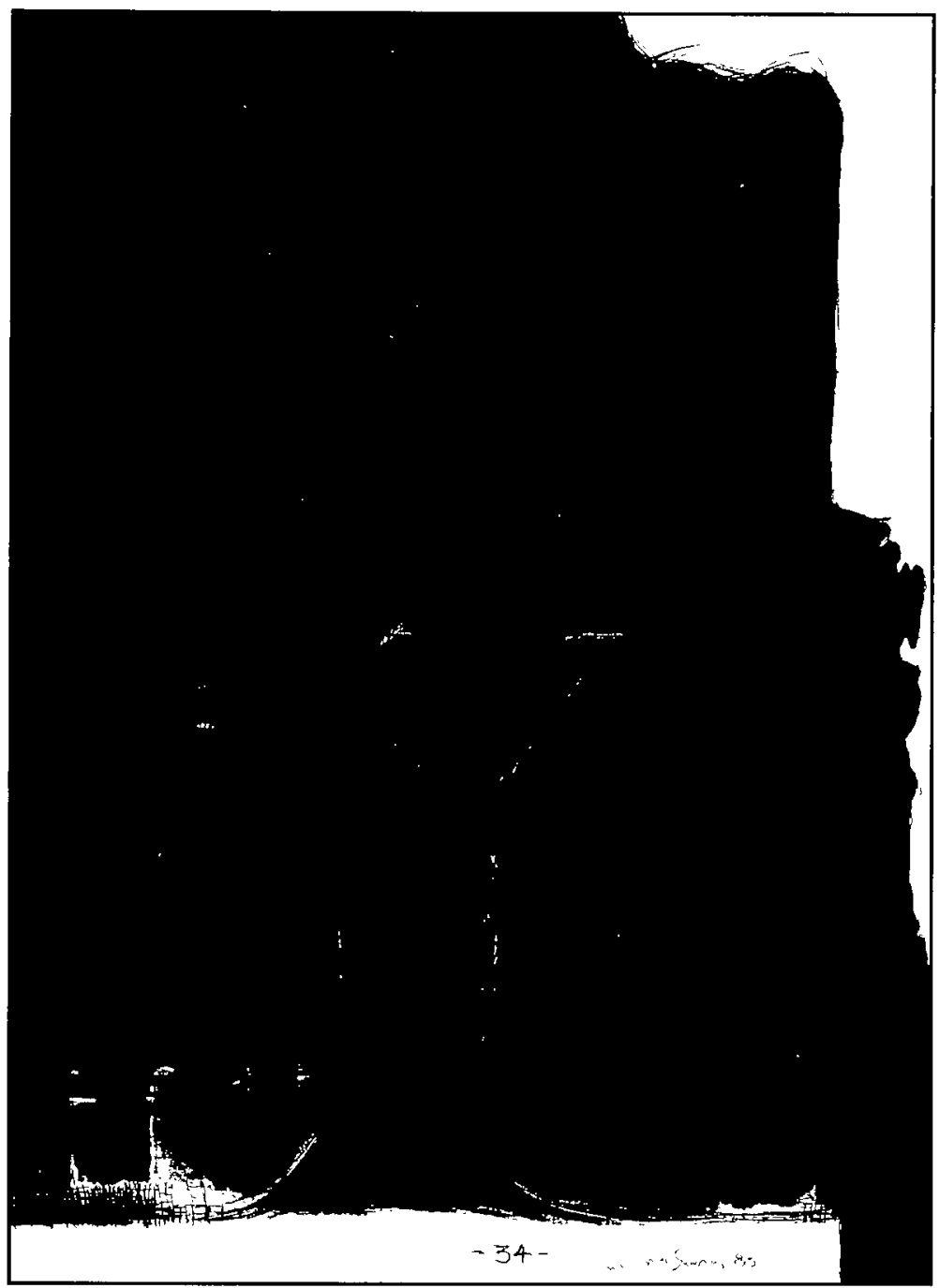

STRANGE WRITINGS ABOUT BERLIN -1988

$120 \times 150 \mathrm{~cm}$

Papier somerset, fusain, pastels, acrylique 